\title{
Macrophages eliminate circulating tumor cells after monoclonal antibody therapy
}

Nuray Gül, 1 Liane Babes, ${ }^{2}$ Kerstin Siegmund ${ }^{2}$ Rianne Korthouwer, ${ }^{1}$ Marijn Bögels, 1,3 Rens Braster, 1 Gestur Vidarsson, ${ }^{4}$ Timo L.M. ten Hagen, ${ }^{5}$ Paul Kubes, ${ }^{2}$ and Marjolein van Egmond ${ }^{1,3}$

\author{
1Department of Molecular Cell Biology and Immunology, VU University Medical Center, Amsterdam, The Netherlands. \\ 2Immunology Research Group, University of Calgary, Calgary, Alberta, Canada. ${ }^{3}$ Department of Surgery, VU University Medical Center, \\ Amsterdam, The Netherlands. " Department of Experimental Immunohematology, Sanquin Research, and Landsteiner Laboratory, \\ Academic Medical Center, Amsterdam, The Netherlands. ${ }^{5}$ Department of Surgery, Section Surgical Oncology, \\ Laboratory of Experimental Surgical Oncology, Erasmus Medical Center, Rotterdam, The Netherlands.
}

\begin{abstract}
The use of monoclonal antibodies (mAbs) as therapeutic tools has increased dramatically in the last decade and is now one of the mainstream strategies to treat cancer. Nonetheless, it is still not completely understood how $\mathrm{mAbs}$ mediate tumor cell elimination or the effector cells that are involved. Using intravital microscopy, we found that antibody-dependent phagocytosis (ADPh) by macrophages is a prominent mechanism for removal of tumor cells from the circulation in a murine tumor cell opsonization model. Tumor cells were rapidly recognized and arrested by liver macrophages (Kupffer cells). In the absence of mAbs, Kupffer cells sampled tumor cells; however, this sampling was not sufficient for elimination. By contrast, antitumor $\mathrm{mAb}$ treatment resulted in rapid phagocytosis of tumor cells by Kupffer cells that was dependent on the high-affinity IgG-binding $\mathrm{Fc}$ receptor $(\mathrm{Fc} \gamma \mathrm{RI})$ and the low-affinity IgG-binding $\mathrm{Fc}$ receptor $(\mathrm{Fc} \gamma \mathrm{RIV})$. Uptake and intracellular degradation were independent of reactive oxygen or nitrogen species production. Importantly, ADPh prevented the development of liver metastases. Tumor cell capture and therapeutic efficacy were lost after Kupffer cell depletion. Our data indicate that macrophages play a prominent role in $\mathrm{mAb}$-mediated eradication of tumor cells. These findings may help to optimize $\mathrm{mAb}$ therapeutic strategies for patients with cancer by helping us to aim to enhance macrophage recruitment and activity.
\end{abstract}

\section{Introduction}

Therapeutic monoclonal antibodies (mAbs), which can be designed to specifically interact with tumor-associated antigens, represent a promising novel category of drugs for targeting malignancies in addition to chemotherapy or radiotherapy $(1,2)$. The anti-CD20 $\mathrm{mAb}$ rituximab was one of the first drugs that was approved for clinical use to treat B cell malignancies (3). Its unprecedented success prompted the development of a multitude of new antitumor mAbs, such as the anti-HER-2 mAb trastuzumab to treat breast carcinoma and the anti-EGFR mAbs cetuximab and panitumumab to treat head and neck cancer and metastasized colorectal carcinoma. The therapeutic mode of action of $\mathrm{mAbs}$ is, however, still incompletely understood and heavily debated, in spite of an overwhelming number of in vitro, in vivo, and patient studies (1-5).

Several direct and indirect mechanisms of mAb therapy have been proposed. Direct mechanisms include the induction of apoptosis, inhibition of proliferation, or sensitization of tumor cells for chemotherapy and likely play an important role in clinical successes of mAb therapy (1-5). For instance, mutations in EGFR signaling pathways in colorectal cancer seriously interfere with therapeutic success of anti-EGFR mAbs (6). Furthermore, most mAbs that are currently used in the clinic are of the IgG1 subclass, which activates the complement cascade through the classical pathway, leading to complement-dependent lysis (CDC). The role of CDC in patients is not yet completely clear. However, it was shown that

Authorship note: Nuray Gül and Liane Babes, as well as Paul Kubes and Marjolein van Egmond, contributed equally to this work.

Conflict of interest: The authors have declared that no conflict of interest exists. Citation for this article: J Clin Invest. 2014;124(2):812-823. doi:10.1172/JCI66776. polymorphisms in the C1QA gene correlated with therapeutic efficacy of rituximab in patients with follicular lymphoma (7). The $\mathrm{Fc}$ region of $\operatorname{IgG}$ additionally interacts with $\mathrm{IgG}$ Fc receptors $(\mathrm{Fc} \gamma$ receptors) that are expressed on immune effector cells. Fcy receptor-mediated mechanisms proved essential for therapeutic efficacy in vivo, since $\mathrm{mAb}$ immunotherapy was ineffective in mice lacking one or more of the activating Fc $\gamma$ receptors Fc $\gamma$ RI, Fc $\gamma$ RIII, or FcyRIV (8-11). When mice were deficient for the inhibitory receptor Fc $\gamma$ RII, antitumor mAb therapy was, by contrast, more effective in preventing tumor development (9). Additionally, strong correlations between success of mAb therapy in patients and $\mathrm{Fc}$ receptor polymorphisms that affect affinity for IgG (Fc $\gamma$ RIIa$131 \mathrm{H} / \mathrm{R}$ and $\mathrm{Fc} \gamma \mathrm{RIII}-158 \mathrm{~V} / \mathrm{F}$ ) have been demonstrated (12-14).

This supports that $\mathrm{F} c \gamma$ receptor-mediated effector functions are essential for therapeutic efficacy of $\mathrm{mAb}$ therapy in patients with cancer. Several Fc $\gamma$ receptor-expressing immune cells have been proposed to execute cytotoxicity during mAb therapy. In general, NK cells are considered as main effector cells, which induce apoptosis in target cells during a process that is referred to as antibody-dependent cellular cytotoxicity (ADCC) (15). Additionally, macrophages have cytotoxic capacity, which can involve diverse mechanisms, including ADCC, release of reactive oxygen species and reactive nitrogen species (ROS and RNS), and antibody-dependent phagocytosis (ADPh) $(16,17)$. Recently, it was demonstrated that neutrophils were required for therapeutic efficacy of $\mathrm{mAbs}$ in a subcutaneous tumor model in mice (18).

Notwithstanding the initial success of treating hematological malignancies with mAbs, therapeutic accomplishments in targeting solid tumors remain somewhat disappointing. This is partly explained by the lack of efficacy when direct effects of mAbs are inhibited. For instance, the anti-HER-2 mAb trastuzumab is 
A
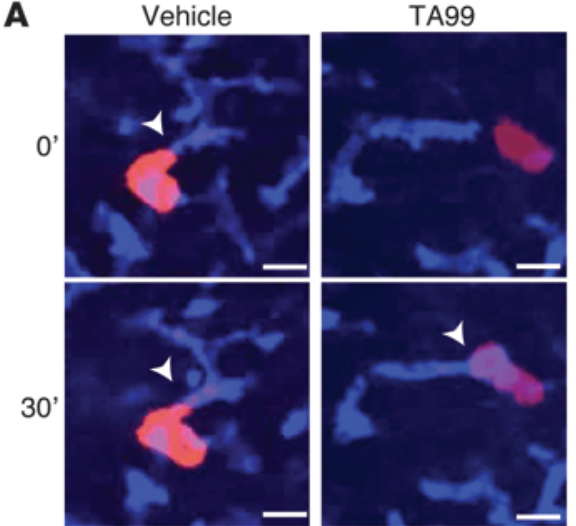

60
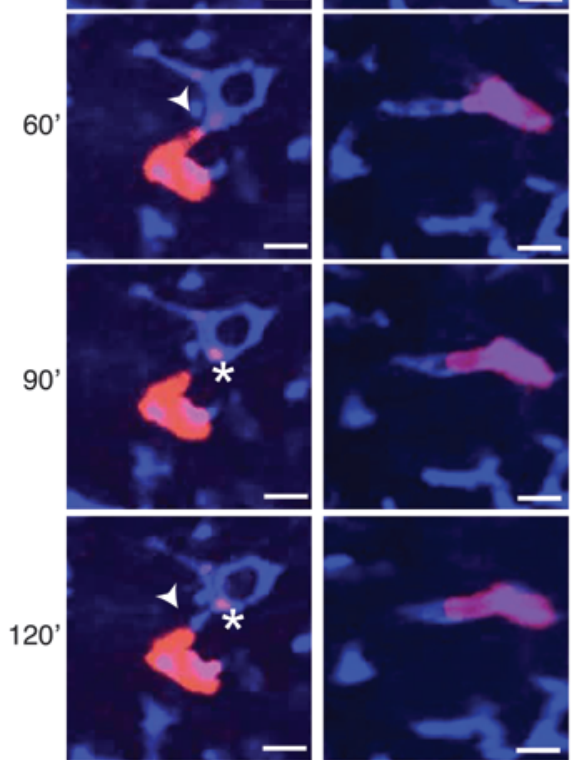

240
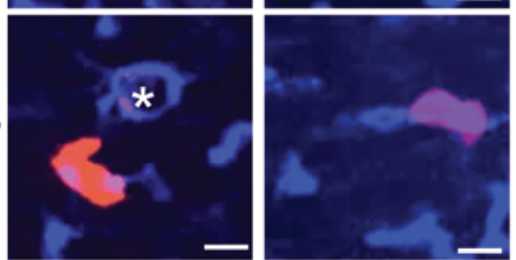

B
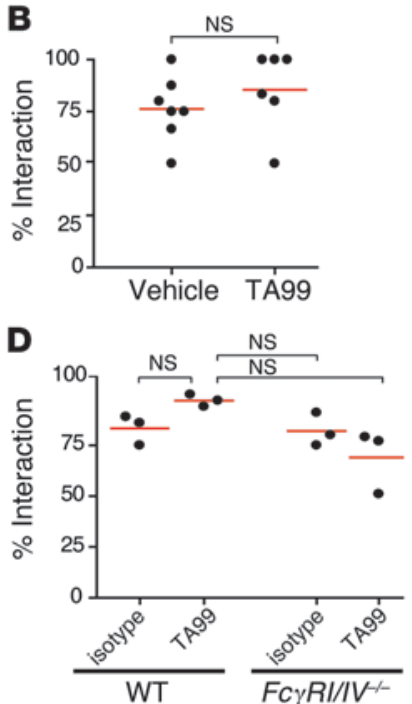

Figure 1

Opsonization with specific mAbs results in rapid phagocytosis of tumor cells by macrophages. (A) Intravital microscopy images in time series (minutes) of B16F10 cells (red) and Kupffer cells (blue) in livers of mice that were treated with either vehicle or TA99 mAbs. Arrowheads indicate contact sites between Kupffer cells and tumor cells. Asterisks indicate uptake of small tumor cell particles. Scale bar: $25 \mu \mathrm{m}$. (B) Percentage of B16F10 tumor cells that interact with Kupffer cells. (C) Percentage phagocytosis of B16F10 tumor cells by Kupffer cells. (D) Percentage of B16F10 tumor cells that interact with Kupffer cells in wild-type mice or $F c \gamma R I / / V^{-1}$ mice. (E) Percentage phagocytosis of B16F10 tumor cells by Kupffer cells in wild-type mice or $F c \gamma R I / I V^{-1-}$ mice. (F and $\left.\mathbf{G}\right)$ In vitro livecell microscopy of DiO-labeled (green) macrophages and Dil-labeled (red) B16F10 cells in the presence of $(\mathbf{F})$ isotype or $(\mathbf{G})$ TA99. Scale bar: $10 \mu \mathrm{m}$. Time points are indicated (minutes). The asterisk in $\mathbf{F}$ indicates tumor cell division. (H) In vitro microscopy images after 24 hours. Scale bar: $50 \mu \mathrm{m}$. Data are representative of at least 3 independent experiments.

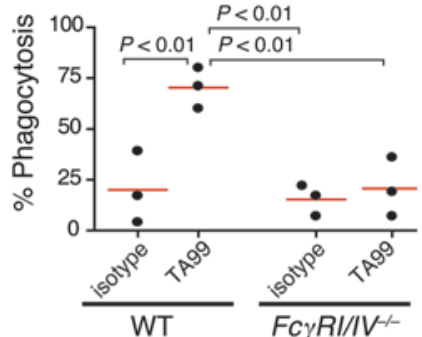

$\mathbf{F}$
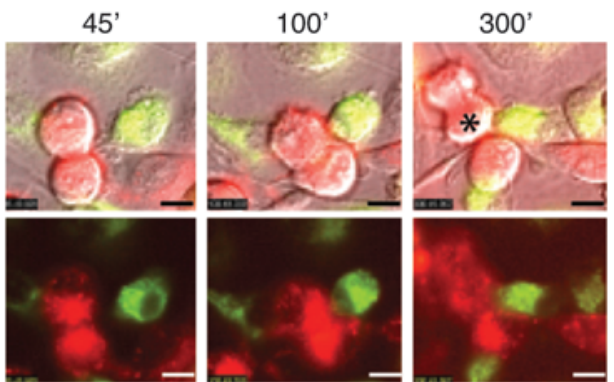

G
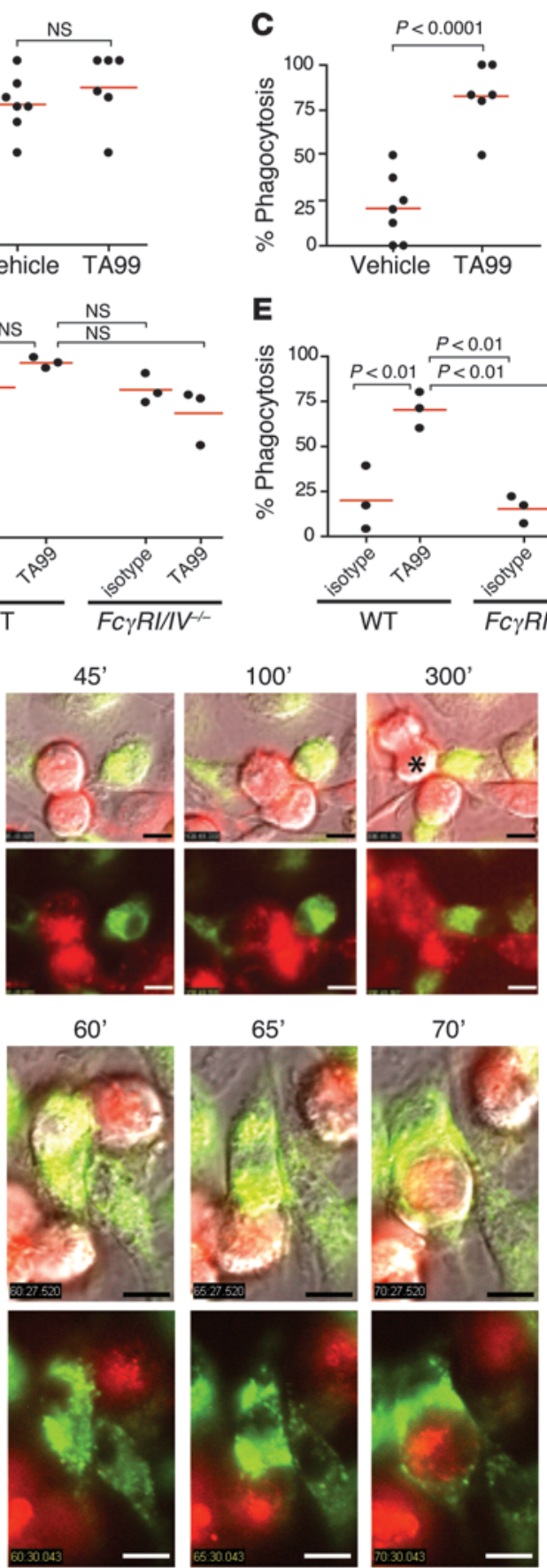

\section{E}

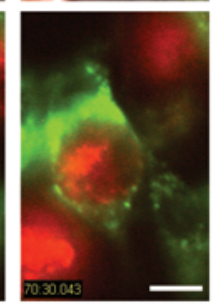

H

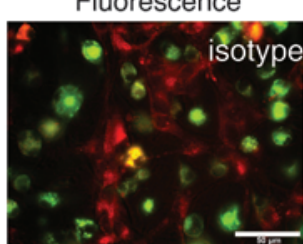

Bright field

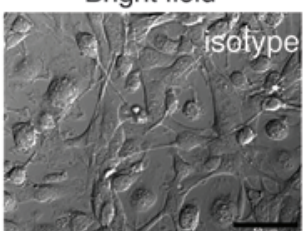

Merge

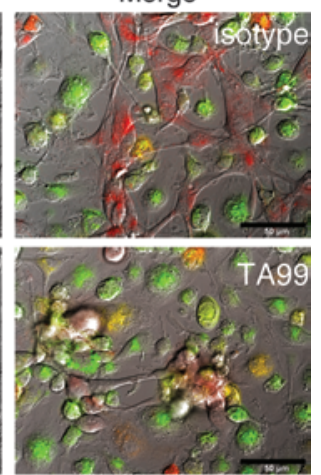


only effective when HER-2 is overexpressed, which is the case in approximately $20 \%$ to $25 \%$ of patients with breast cancer, whereas anti-EGFR mAb therapy is only effective when tumors express wild-type KRAS (6). Direct mechanisms of mAbs are fully dependent on the biology of the target antigen and, as such, are difficult to influence. Fc $\gamma$ receptor-mediated functions are, however, mechanisms that can be improved upon. Understanding the precise mode of action(s) of mAb therapy will contribute to optimization of $\mathrm{mAb}$ therapeutical strategies to improve efficacy in these patients.

We previously identified a potential novel mAb-based therapeutic strategy for patients undergoing resection of primary colorectal cancer $(11,19)$. We demonstrated that surgery paradoxically enhances the risk of liver metastases development by promoting adherence of circulating tumor cells $(20,21)$. Importantly, presurgical $\mathrm{mAb}$ therapy prevented liver metastases outgrowth in animals, which was depended on the presence of Fc $\gamma \mathrm{RI}$ and Fc $\gamma \mathrm{RIV}$ $(11,19)$. This supported the involvement of liver macrophages (Kupffer cells), because both receptors are expressed by the myeloid mononuclear network in mice. Furthermore, approximately $80 \%$ of tumor cells were in contact with Kupffer cells in the absence of tumor-specific mAb, which was increased to approximately $90 \%$ after mAb treatment (19). Surprisingly, this modest difference completely prevented liver metastases development. To study the exact mechanisms of mAb therapy, we therefore used in vitro live-cell imaging and intravital microscopy of the livers of mice to visualize the mode of action in more detail.

\section{Results}

Phagocytosis of tumor cells by macrophages is dependent on opsonization with specific $m A b$ s and presence of $F c \gamma R I$ and $F c \gamma R I V$. The B16F10 cell line is the only syngeneic murine solid tumor cell line for which a specific $\mathrm{mAb}$ is available (TA99; anti-gp75), and it has previously been used as model system to study antibody therapy of cancer (8, 11). C57BL/6 mice were inoculated with B16F10 cells and treated with either vehicle or TA99 $\mathrm{mAb}$. Intravital imaging in the livers of vehicle-treated mice showed that Kupffer cells were able to interact with and sample small parts of tumor cells, which, however, did not result in elimination of tumor cells (Figure 1A and Supplemental Videos 1 and 2; supplemental material available online with this article; doi:10.1172/JCI66776DS1). In contrast, tumor cells were rapidly recognized and phagocytosed by Kupffer cells in the livers of TA99-treated mice (Figure 1A and Supplemental Videos 1 and 2). Thus, although no difference was observed between tumor cell numbers that were in contact with Kupffer cells after vehicle or TA99 mAb therapy (Figure 1B), the number of tumor cells that had been phagocytosed was significantly increased after TA99 treatment (Figure 1C). To exclude the possibility of nonspecific phagocytosis due to injection of mAbs, experiments were repeated with an isotype control $\mathrm{mAb}$ (murine IgG2a against rat colon cancer cells). Similar results were observed compared to injection of vehicle alone, as Kupffer cells of isotype-treated mice were in contact with tumor cells but unable to phagocytose whole tumor cells (Supplemental Video 3).

We previously demonstrated that prevention of liver metastases outgrowth after antibody treatment was dependent on the expression of Fc $\gamma$ RI and Fc $\gamma$ RIV (11). To investigate whether ADPh by Kupffer cells was dependent on these receptors, we next repeated intravital experiments with Fc $\gamma \mathrm{RI}$-deficient mice. Additionally, the anti-Fc $\gamma$ RIV blocking antibody 9E9 was injected to block inter- action of the therapeutic mAb TA99 with FcRIV (these mice are referred to as $F c \gamma R I / I V^{-/-}$mice). Kupffer cells of $F c \gamma R I / I V^{-/-}$mice were still able to scan and sample tumor cells, comparable to Kupffer cells in livers of wild-type mice (Figure 1D). No difference in the percentage of tumor cells in contact with Kupffer cells was observed after either isotype or TA99 mAb treatment. However, the capacity of Kupffer cells to phagocytose TA99-opsonized tumor cells was lost in $F c \gamma R I / I V^{-/-}$mice (Figure 1E). Thus, ADPh by Kupffer cells required functional $\mathrm{F} c \gamma \mathrm{RI}$ and $\mathrm{Fc} \gamma \mathrm{IV}$ expression.

Prolonged macrophage-tumor cell contact in the presence of an isotype control $\mathrm{mAb}$ was observed in vitro live-cell experiments as well, comparable to that in intravital microscopy experiments. However, macrophages were unable to prevent tumor cell growth (Figure 1F and Supplemental Video 4). Addition of TA99 mAbs resulted in rapid phagocytosis and elimination of tumor cells (Figure 1, G and H, and Supplemental Video 4). Uptake of tumor cells was associated with phagosome-lysosome fusion, which led to rapid acidification, but slow degradation of tumor cells in vitro (Figure 2, A and B, and Supplemental Video 5). This was also observed in intravital studies. When livers of mice were studied 24 hours after injection of B16F10 cells, tumor cells had been degraded into smaller particles in livers of animals that had been treated with TA99, but tumor material was still present (Figure 2, C-E, and Supplemental Video 6). Some small tumor particles were observed in Kupffer cells of vehicle- or isotype-treated mice, but most tumor cells were present in larger clusters, indicative of tumor cell outgrowth (Figure 2, D and E; Supplemental Video 6; and data not shown).

ROS or RNS are not required for $A D P h$. Uptake of $\mathrm{mAb}$-opsonized tumor cells led to production of ROS by Kupffer cells in vivo (Figure 3A). When mice were treated with the ROS scavenger, edaravone (EDA), and the NO synthase inhibitor, $N^{\mathrm{G}}$-monomethyl-L-arginine (L-NMMA), ROS production was absent, but ADPh was unaffected (Figure 3B). No differences in interactions of tumor cells with Kupffer cells were observed when mice were treated with TA99 $\mathrm{mAb}$ alone or when they also received treatment with EDA, L-NMMA, or a combination of EDA and L-NMMA (Figure 3C). Similarly, treatment with EDA, L-NMMA, or a combination of EDA and L-NMMA did not reduce phagocytosis of tumor cells by Kupffer cells after TA99 mAb therapy (Figure 3D). In vitro experiments confirmed that EDA was effective in inhibition of ROS production by macrophages (Figure 3E). However, neither the level, nor the kinetics of ADPh were altered when ROS was inhibited, as phagocytosis of TA99-opsonized tumor cells by macrophages after 1 or 4 hours of coincubation was unaffected by EDA treatment (Figure 3F). Minimal phagocytosis was observed in the presence of an isotype control (data not shown). Similar results were observed with live-cell microscopy experiments. No differences in uptake of TA99-opsonized B16F10gp75 cells, acidification of lysosomes, or degradation were observed when ROS production by macrophages was blocked by incubation with EDA (Figure 3, G-J).

Kupffer cells are the main effector cells in antibody-mediated prevention of tumor outgrowth in the liver. To investigate whether other nonKupffer cell-dependent killing also was occurring, we depleted Kupffer cells with clodronate liposomes (22) prior to injection of tumor cells and mAb. Treatment with TA99 was ineffective when Kupffer cells had been depleted, as large tumor cell clusters were observed 24 hours after tumor cell injection in both vehicle- and TA99 mAb-treated mice (Figure 4A). Additionally, there was no difference in size of tumor cell particles between vehicle- or TA99- 
A
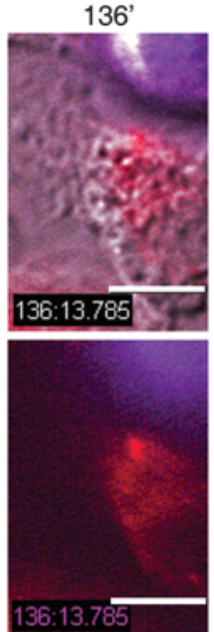

8

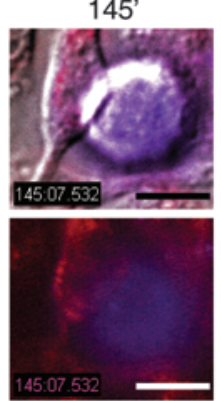

c

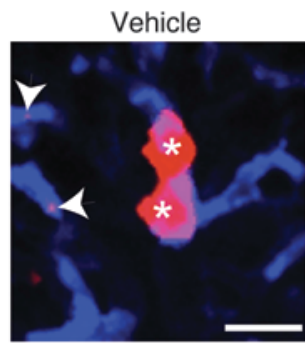

171
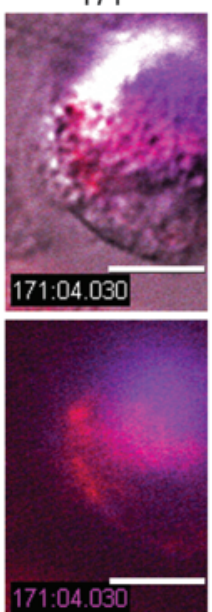

184
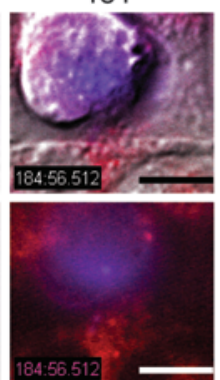

205
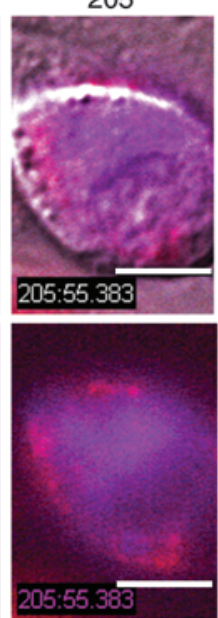

$219^{\prime}$
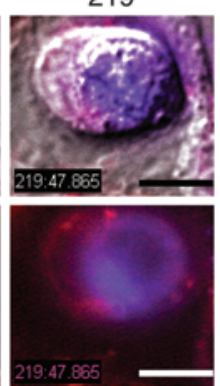

TA99

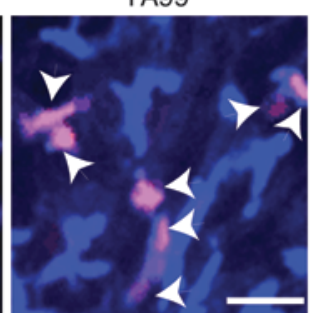

$285^{\prime}$
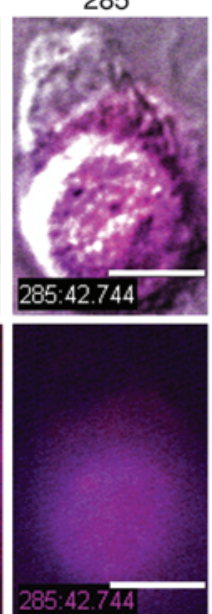

$419^{\prime}$

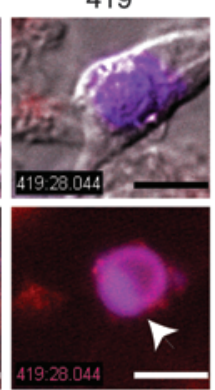

D

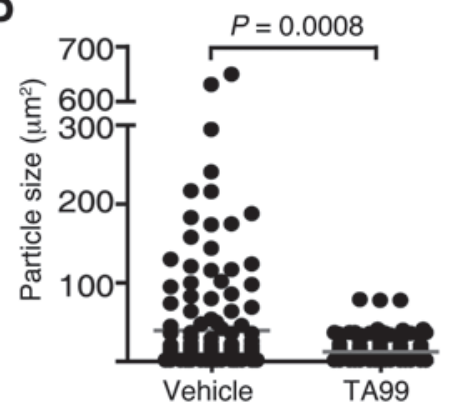

690
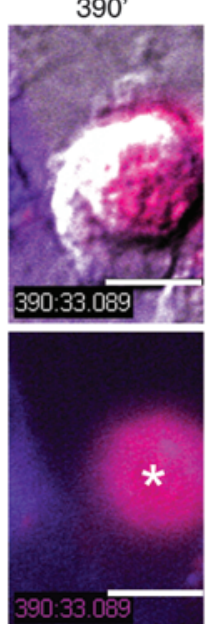

$614^{\prime}$
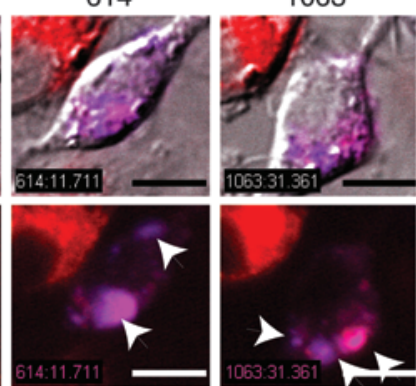

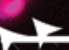

1063

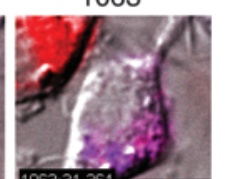

Scale bar: 10 um. (C) Kupffer cells (blue) and B16F10 cells (red) observed in vivo by intravital microscopy 24 hours after injection of tumor cells in livers of vehicle- or TA99-treated mice. Scale bar: $25 \mu \mathrm{m}$. Arrowheads indicate tumor cell particles within Kupffer cells. Asterisks indicate whole tumor cells. (D) Size of tumor cell particles in livers of vehicle- or TA99-treated mice. (E) 3D reconstruction of Kupffer cells (blue) and B16F10 cells (red) in vehicle- or TA99treated mice. Colors are artificial. Scale bar: $50 \mu \mathrm{m}$. Data are representative of 3 independent experiments.
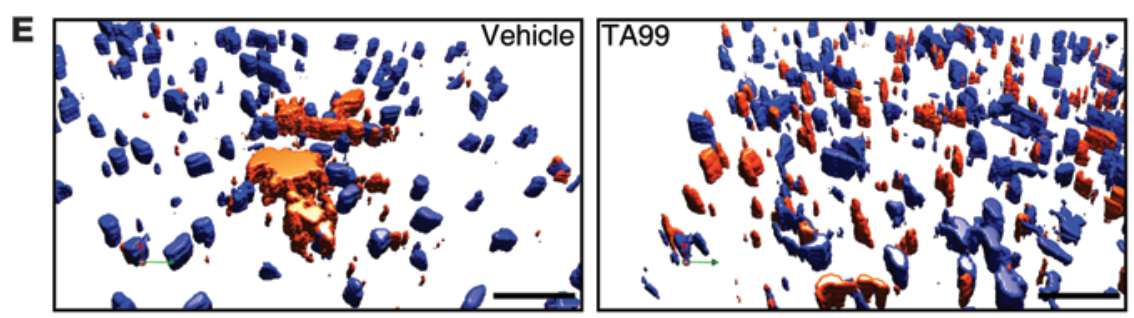

treated mice (Figure 4B). Moreover, whereas treatment with TA99 almost completely prevented outgrowth of liver metastases after 3 weeks in control mice, no therapeutic protection was observed in Kupffer cell-depleted mice (Figure 4C). Thus, these results suggest that Kupffer cells are essential in the arresting and killing of tumor cells in the liver after mAb therapy and that direct killing by antibody-induced apoptosis or CDC are less likely mechanisms of action. We therefore investigated the distribution of tumor cells after TA99 mAb therapy in control and Kupffer cell-depleted mice. Whereas no tumor cells were found in blood of control mice after TA99 mAb therapy, the number of circulating tumor cells was increased in Kupffer cell-depleted mice (Figure 5A). Furthermore, increased numbers of tumor cells were found in spleens and lungs of Kupffer cell-depleted mice (Figure 5, B and C), supporting that Kupffer cells play a prominent role in arresting circulating tumor cells after mAb therapy.

The anti-EGFR $m A b$ induces $A D P h$ and prevents micrometastasis formation. The B16F10 cell line is an excellent model system to study $\mathrm{mAb}$ therapy in a syngeneic model, as it will not trigger immune responses against foreign (human) antigens. However, to investi- 
A
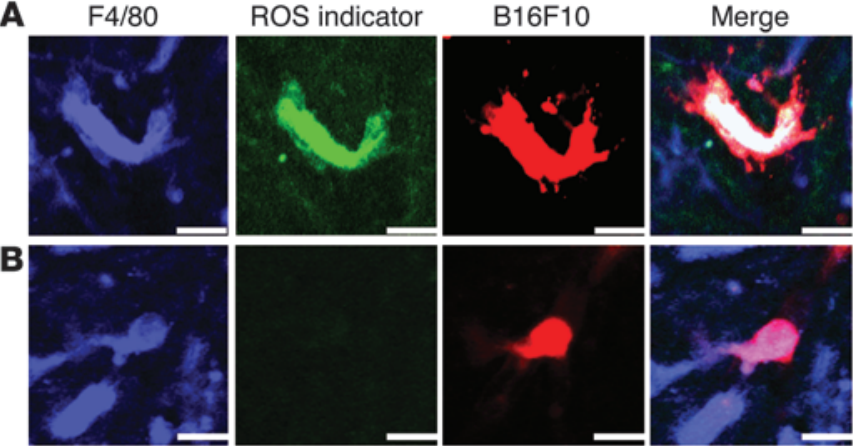

C
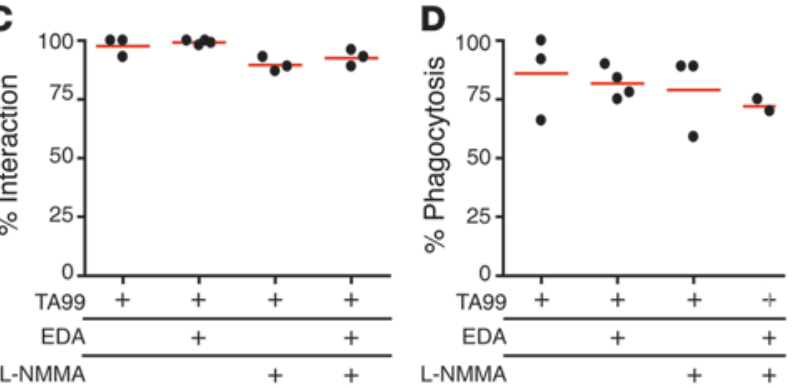

L-NMMA

E

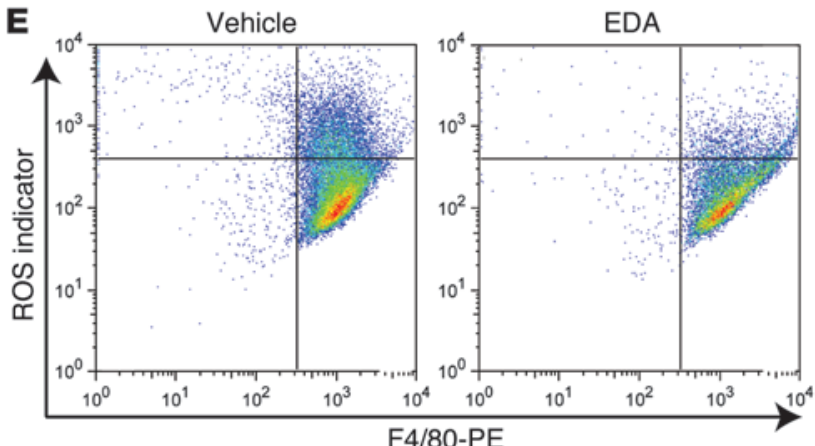

F4/80-PE

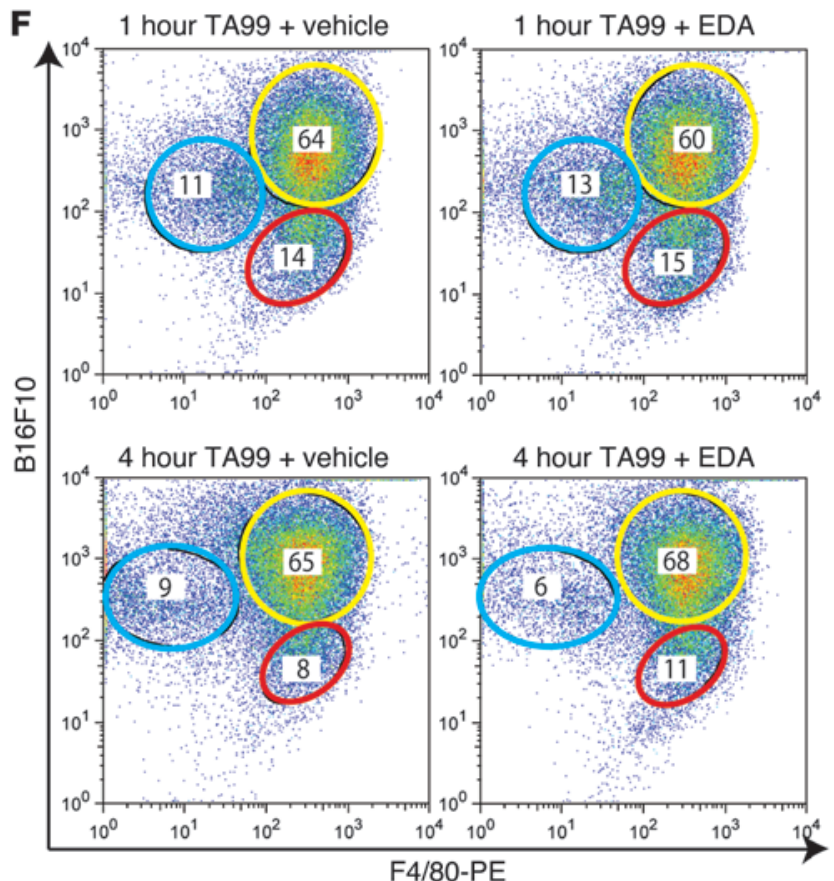

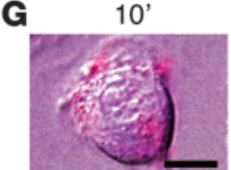
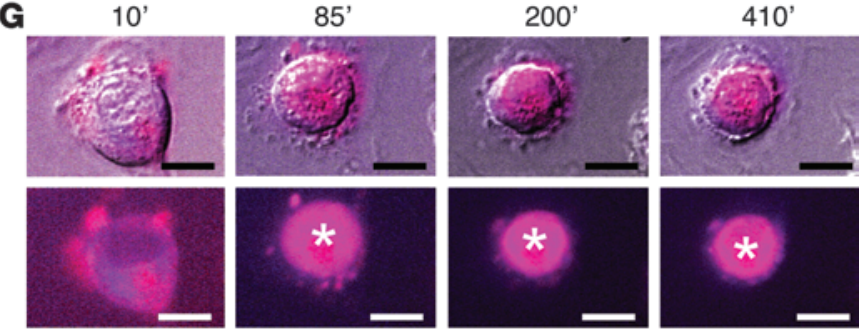

H
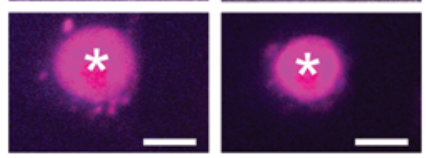

200'
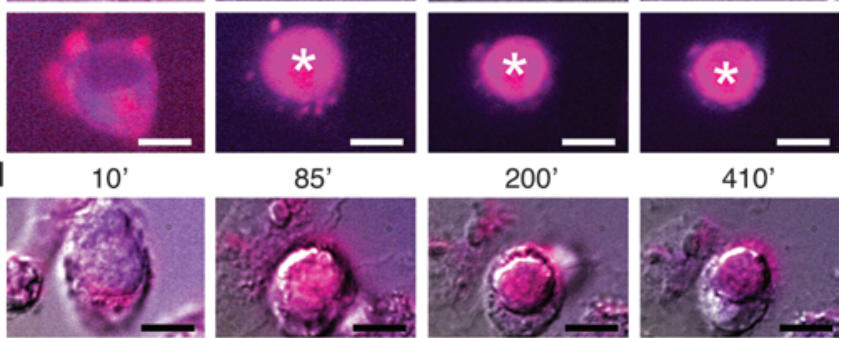

410

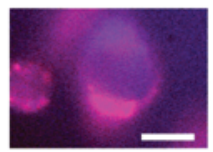

I

10
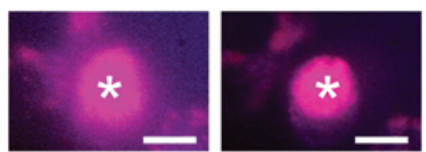

85
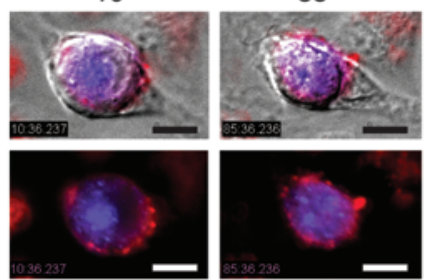

J

10

100 '
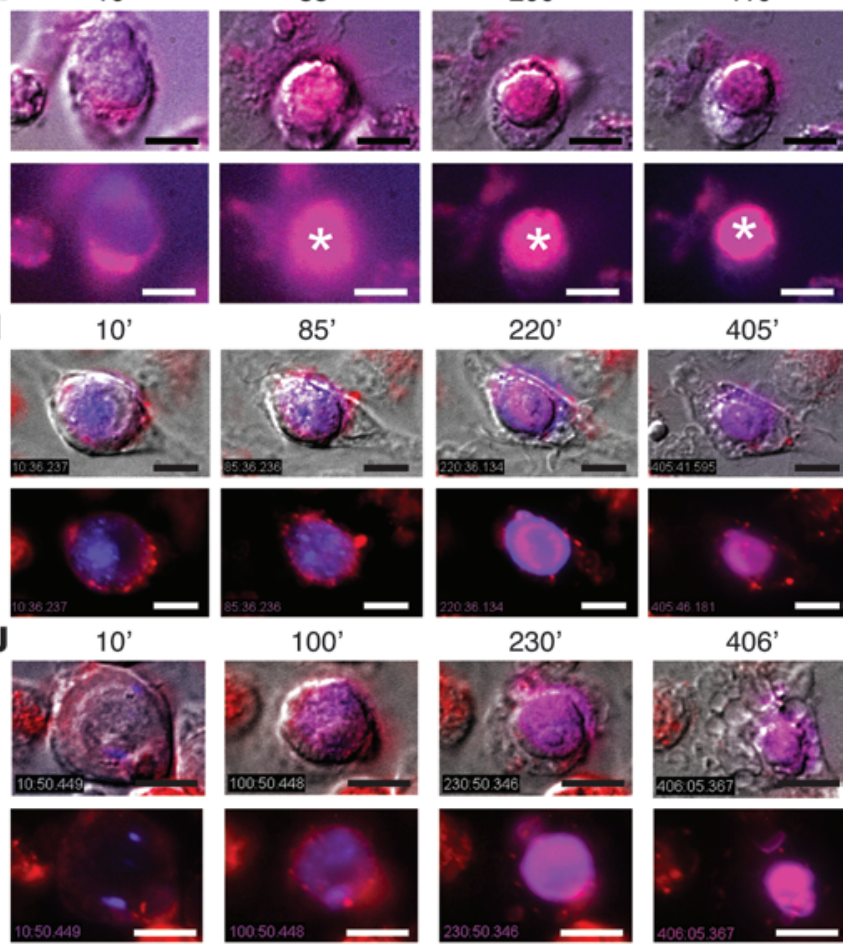

405
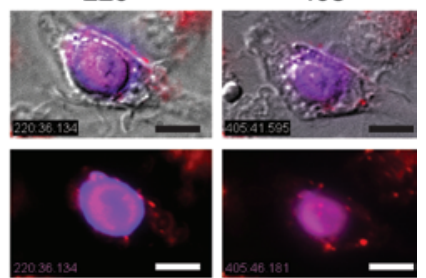

230

406
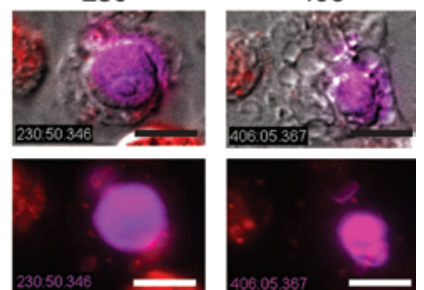

Figure 3

ROS or RNS are not required for ADPh. (A and B) Formation of ROS after ADPh of opsonized tumor cells by Kupffer cells in livers of mice treated with (A) TA99 or (B) TA99, EDA, and L-NMMA. Scale bar: $25 \mu \mathrm{m}$. (C) Percentage of tumor cells interacting with Kupffer cells and (D) percentage phagocytosis of tumor cells by Kupffer cells in the livers of mice that were treated with TA99, TA99 and EDA, TA99 and L-NMMA, or TA99, EDA, and L-NMMA. (E) ROS production by macrophages after stimulation with $50 \mu \mathrm{g} / \mathrm{ml}$ peptidoglycan with and without EDA. Gates in the top right corners indicate ROS-producing macrophages. (F) Macrophages were incubated for 1 or 4 hours with B16F10-DiB cells and TA99 in the absence or presence of EDA. Macrophages were stained with F4/80-PE. Yellow circles denote double-positive cells (indicative of phagocytosis); red circles denote $\mathrm{F} 4 / 80-\mathrm{PE}^{+}$macrophages; and blue circles denote nonphagocytosed B16F10-DiB cells. Numbers indicate percentage of total cells. (G-J) Phagocytosis of DiB-labeled (blue) B16F10gp75 cells by ( $\mathbf{G}$ and $\mathbf{H}$ ) LysolD-labeled (red) or (I and $\mathbf{J})$ Dil-labeled (red) macrophages in the presence of ( $G$ and I) TA99 or ( $\mathbf{H}$ and $\mathbf{J}$ ) TA99 and EDA. Asterisks in $\mathbf{G}$ and $\mathbf{H}$ indicate the phagolysosome. Time points are indicated (minutes). The top rows show overlay of bright field and fluorescence, and the bottom rows show fluorescence only. Scale bar: $10 \mu \mathrm{m}$. 
A

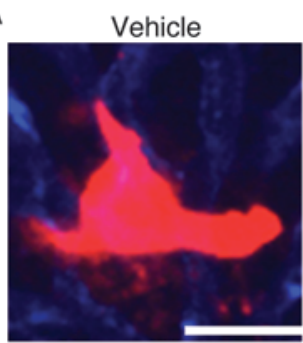

TA99

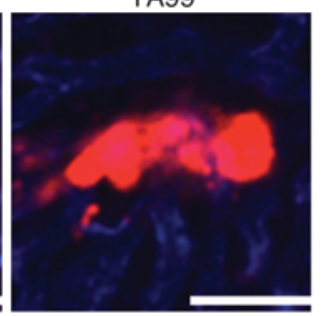

B

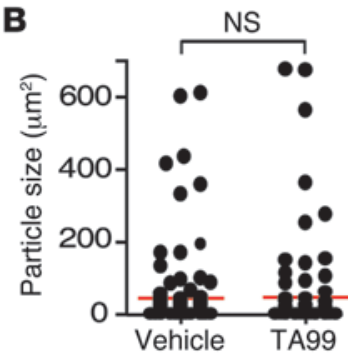

C

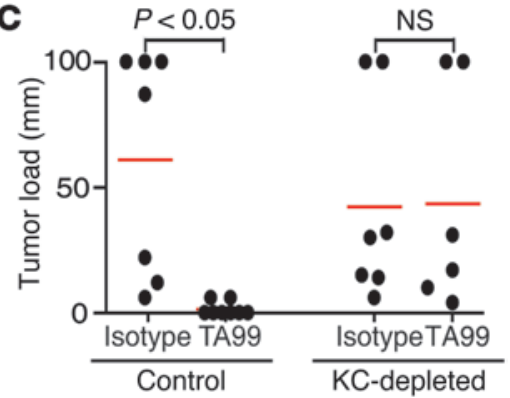

Figure 4

Kupffer cells are the main effector cells in mAb-mediated elimination of tumor cells. (A) B16F10 cells (red) 24 hours after treatment with either vehicle or TA99 in livers of Kupffer cell-depleted mice visualized by intravital microcopy. Scale bar: $50 \mu \mathrm{m}$. (B) Size of tumor cell particles 24 hours after vehicle or TA99 treatment in Kupffer cell-depleted mice. (C) Liver metastases development after 3 weeks in control or Kupffer cell-depleted mice that were treated with isotype control or TA99 antibody. Data are representative of 3 independent experiments. KC, Kupffer cell.

gate a more clinically relevant situation, we next transfected the murine colon carcinoma cell line C26 with human EGFR (C26hEGFR cells) and determined whether the anti-EGFR mAb cetuximab - which is used clinically to treat metastatic colon carcinoma (23) - could prevent liver metastases development. Similar to the B16F10 model, we observed increased phagocytosis of anti-EGFR mAb-opsonized C26-hEGFR cells, compared with vehicle-treated tumor cells (Figure 6, A and B). In contrast to cells that were nonopsonized (vehicle-treated), tumor cells that were coated with antiEGFR mAb were recognized and taken up by Kupffer cells (Figure 6C). However, anti-EGFR mAb-opsonized tumor cells grew rapidly in Kupffer cell-depleted mice (Figure 6D). Importantly, 4 and
5 days after injection of tumor cells, we observed more and larger micrometastases in the livers of control mice that received vehicle treatment or Kupffer cell-depleted mice after anti-EGFR mAb treatment, compared with livers of wild-type mice after anti-EGFR mAb treatment (Figure 6, E-H). Thus, similar to results with TA99, these data indicate that Kupffer cells are the major effector immune cell type in tumor-specific antibody-mediated prevention of tumor development by eliminating circulating tumor cells.

To investigate whether anti-EGFR mAbs could eradicate established liver metastases, tumor-bearing mice were treated with either vehicle or anti-EGFR mAb. However, tumor burden was similar in both groups (Supplemental Figure 1), suggesting that

\section{Figure 5}

Kupffer cells are essential in arresting tumor cells in the liver after TA99 mAb therapy. (A) Representative FACS scatter plots of circulating tumor cells in control or Kupffer cell-depleted mice after TA99 $\mathrm{mAb}$ therapy and quantification of tumor cells in blood from control and Kupffer celldepleted mice. SSC, side scatter. (B and C) Representative images from (B) lungs and (C) spleens of control and Kupffer celldepleted mice and quantification of numbers of tumor cells per field of view (fov). Arrowheads indicate red tumor cells. Blue, cell nuclei; red, B16F10; green, F4/80+ macrophages. (Note that alveolar macrophages express very low levels of $\mathrm{F} 4 / 80$; ref. 48.) Scale bar: $50 \mu \mathrm{m}$.
A

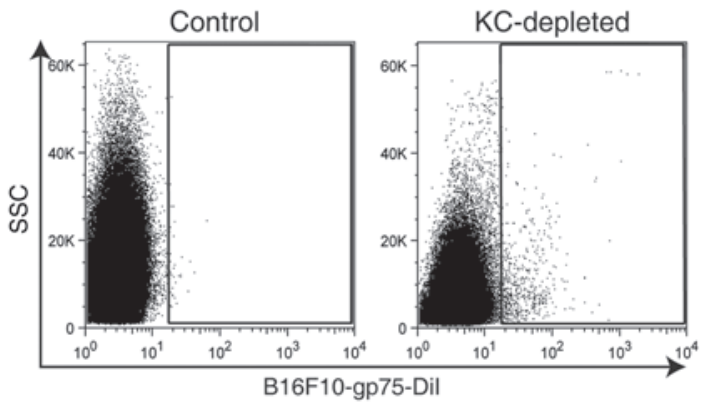

B
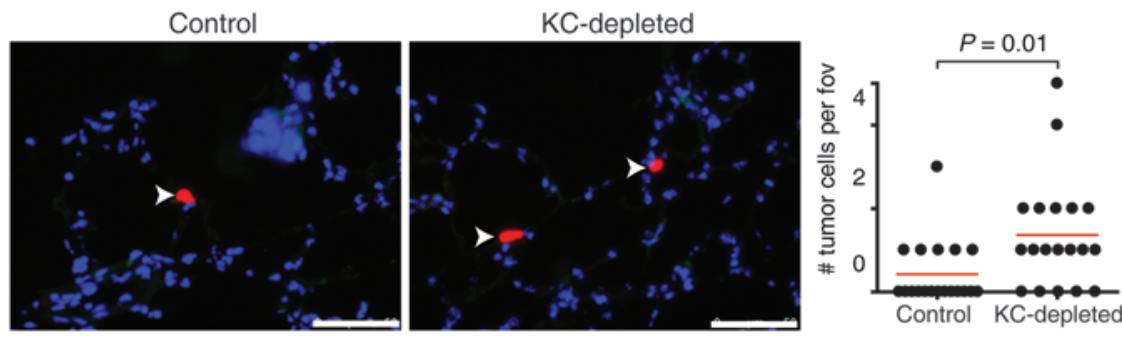

C

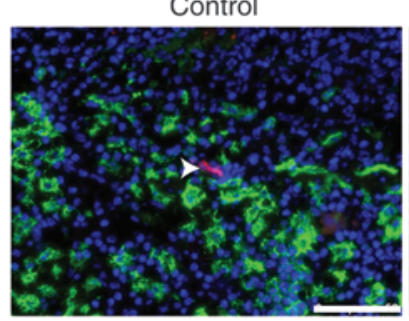

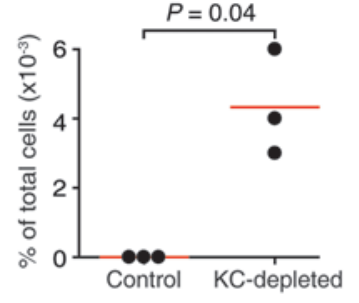

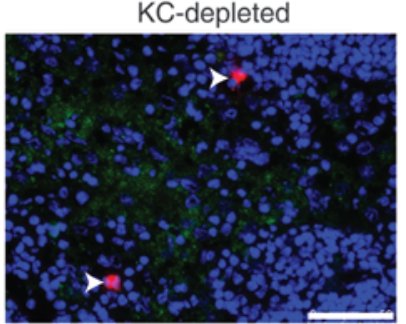

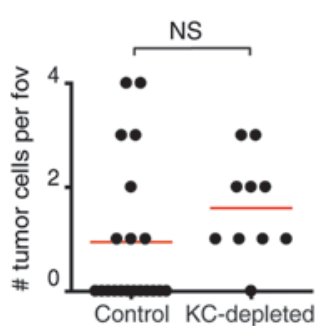


A

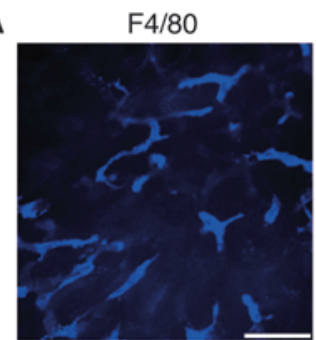

B

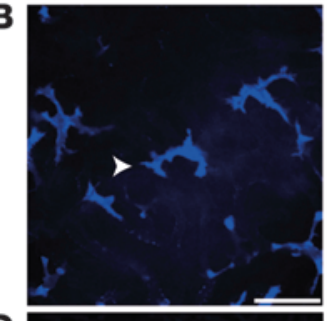

D

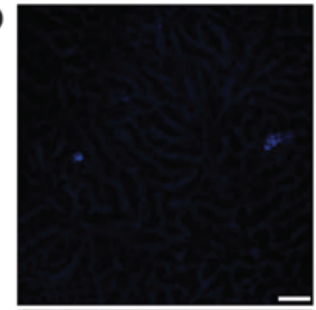

E

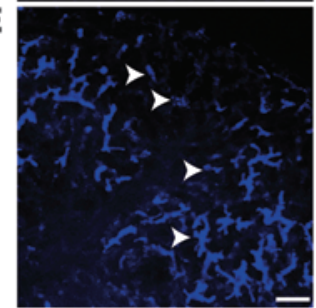

C26-hEGFR-Dil
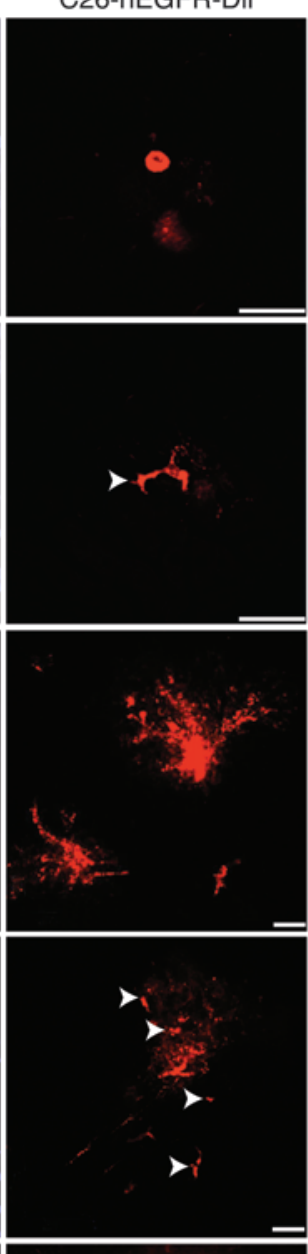
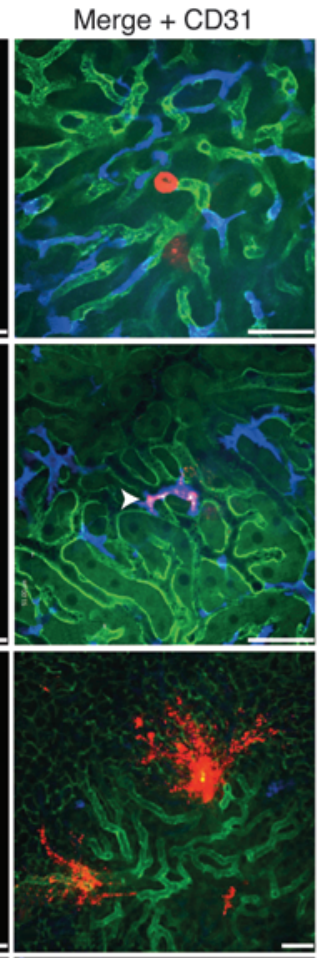

F

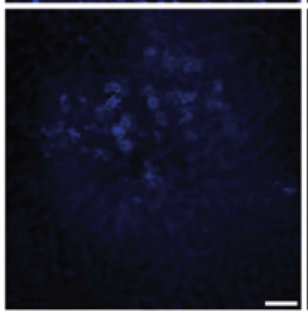

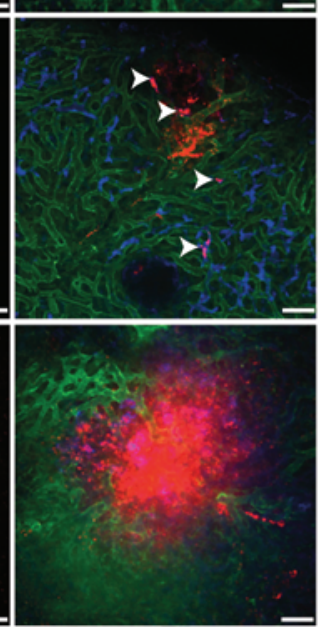
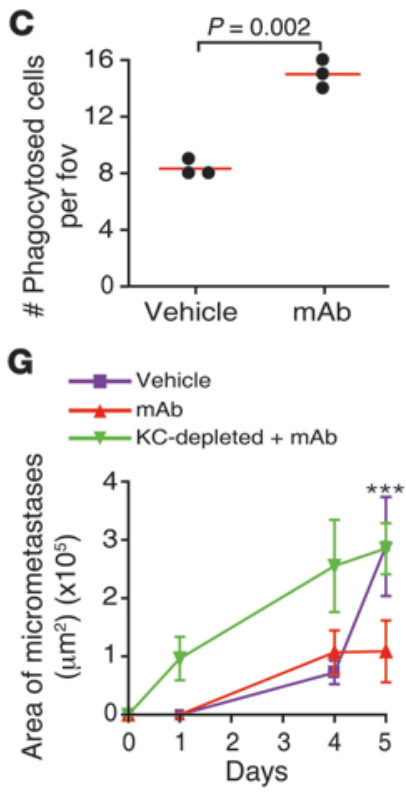

H

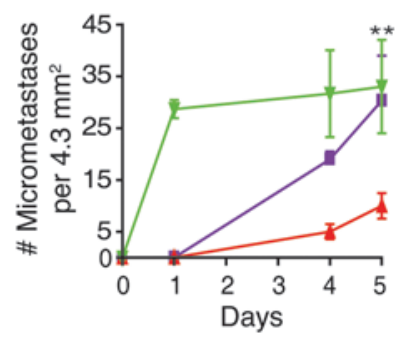

\section{Figure 6}

Anti-EGFR mAbs reduce outgrowth of C26-hEGFR liver metastases. (A-D) Kupffer cell-dependent phagocytosis of tumor cells in sinusoids of the liver 1 day after inoculation. Representative images of livers (A) in mice receiving vehicle or (B and $\mathbf{D}$ ) anti-EGFR mAb-opsonized C26-hEGFR cells and (D) in Kupffer cell-depleted mice. (C) Number of phagocytosed tumor cells per field of view in livers of mice that received vehicle or antiEGFR mAb-opsonized C26-hEGFR cells. (E-H) Reduced formation of micrometastases and outgrowth in the livers of mice is Kupffer cell dependent. ( $E$ and $\mathbf{F}$ ) Representative images from livers of $(\mathbf{E})$ control or $(\mathbf{F})$ Kupffer cell-depleted mice, which received anti-hEGFR mAb-opsonized C26-hEGFR cells, 4 days after injection of tumor cells. (G and $\mathbf{H}$ ) Area and number of micrometastases in livers of control or Kupffer cell-depleted mice, which were either injected with vehicle or anti-EGFR mAb-coated tumor cells. Arrowheads indicate uptake of tumor cells by Kupffer cells. Blue, F4/80+ Kupffer cells; red, C26-hEGFR-Dil; green, CD31. Scale bar: $50 \mathrm{~mm}$. ${ }^{* \star} P<0.01,{ }^{\star \star *} P<0.001$ vehicle vs. anti-EGFR mAbs.

anti-EGFR $\mathrm{mAb}$ is ineffective in eliminating existing liver metastases. This may have been due to the inability of anti-EGFR $\mathrm{mAb}$ to recruit Kupffer cells, which were stationary (data not shown).

Additionally, live-cell imaging experiments demonstrated that C26-hEGFR cells were phagocytosed by macrophages in the presence of anti-EGFR $\mathrm{mAb}$ (but not in the presence of an isotype control; data not shown). Similar to results with B16F10gp75 cells, we observed neither differences in uptake of C26-hEGFR in the presence of anti-EGFR mAbs nor in acidification of lysosomes and degradation when ROS production by macrophages was blocked by incubation with EDA (data not shown). Moreover, experiments were repeated with a human epithelial carcinoma cell line (A431 cells). Phagocytosis of A431 tumor cells was increased substantially by incubation with macrophages and anti-EGFR mAb. No major differences in uptake of anti-EGFR mAb-opsonized A431 cells or acidification of lysosomes were observed when ROS pro- 
A

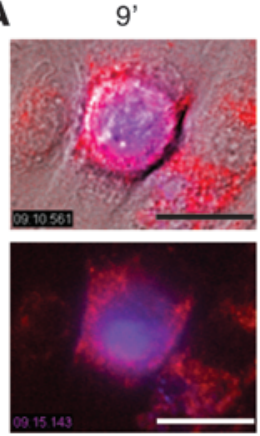

B
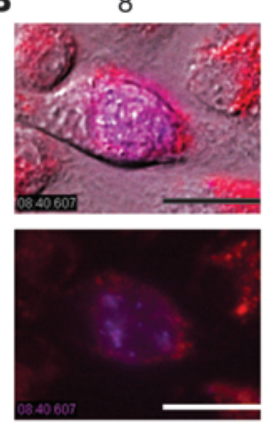

C

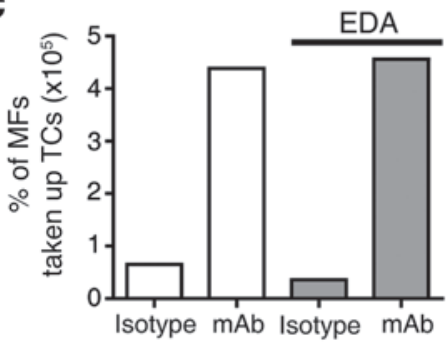

D

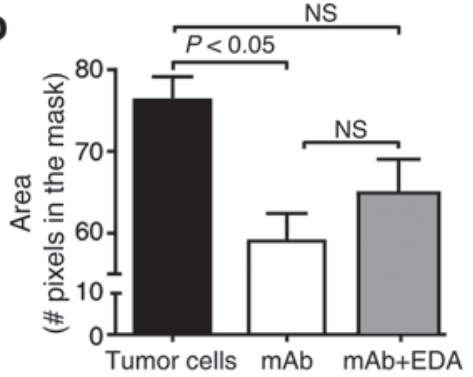

39

38

EDA
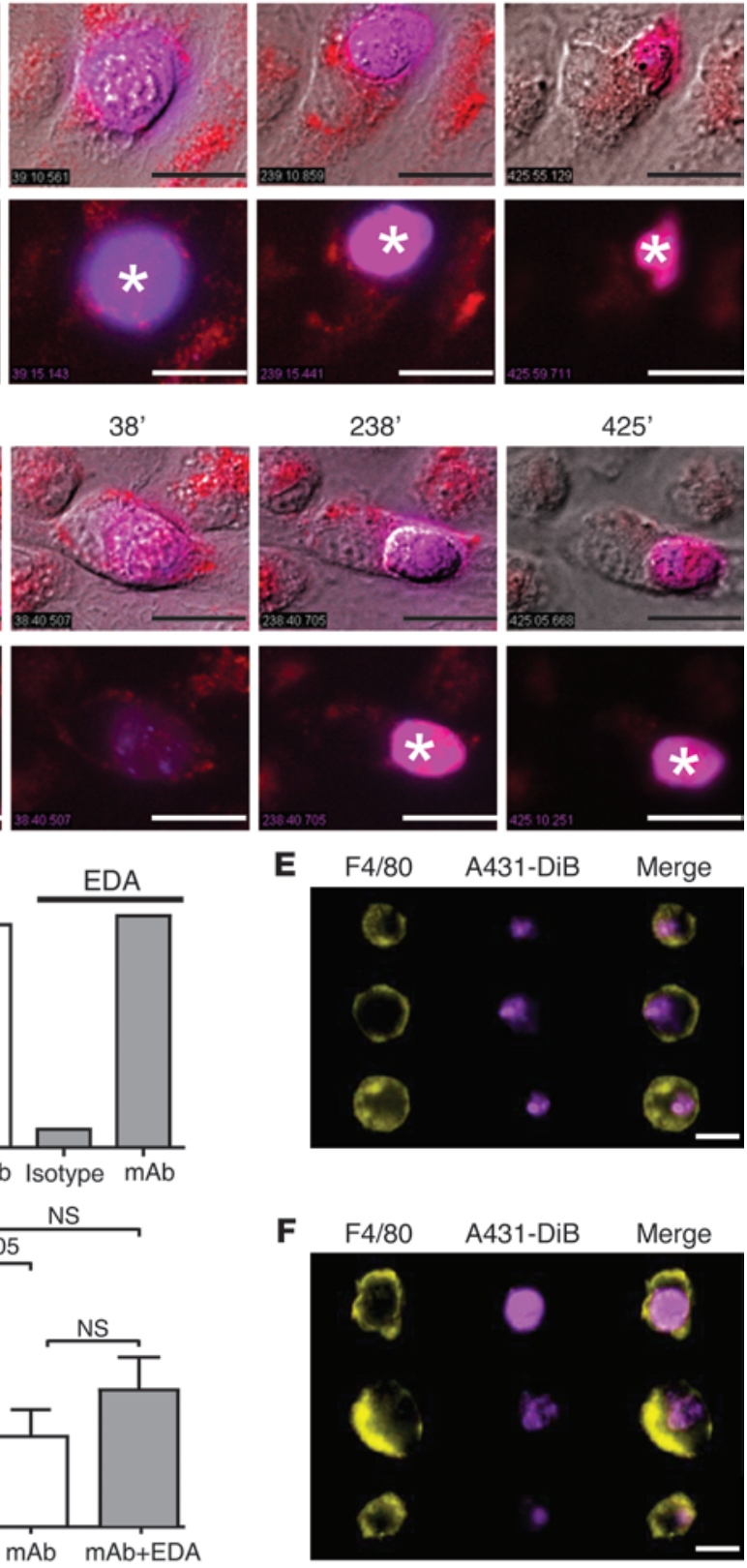

Figure 7

Anti-EGFR mAbs induce ADPh of human epithelial carcinoma A431 cells by macrophages. (A and B) Phagocytosis of DiB-labeled (blue) B16F10gp75 cells by LysolD-labeled (red) macrophages in the presence of (A) anti-EGFR mAbs or (B) anti-EGFR mAbs and EDA. Asterisks indicate the phagolysosome. The top rows show overlay of bright field and fluorescence, and the bottom rows show fluorescence only. Scale bar: $20 \mu \mathrm{m}$. (C) Percentage of macrophages that have phagocytosed $A 431$ cells in the presence of an isotype or anti-EGFR mAb with or without EDA. MF, macrophage; TC, tumor cell. (D) Quantification of tumor cell size. (E and $\mathbf{F}$ ) Representative images of anti-EGFR mAb-opsonized A431 tumor cells that have been phagocytosed and degraded by $(\mathbf{E})$ control macrophages or (F) EDA-treated macrophages. Scale bar: $14 \mu \mathrm{m}$

with EDA, compared with untreated macrophages, which suggests that ROS may play a minor role in degradation of intracellular tumor cells.

\section{Discussion}

To date, modes of action of mAbs have been proposed based on in vitro results or experiments with mice that were either deficient for specific components of the immune system or in which immune components or cells had been depleted (8-11, 19, $24,25)$. With the use of intravital microscopy, we now demonstrate for the first time directly that $\mathrm{mAb}$ therapy induces phagocytosis of circulating tumor cells by macrophages. This was dependent on Fc $\gamma$ RI and Fc $\gamma$ RIV, which is in accordance with earlier studies in which we demonstrated that $\mathrm{F}_{\mathrm{C}} \gamma \mathrm{RI}$ and $\mathrm{F} c \gamma \mathrm{RIV}$ were necessary for the prevention of liver metastases after mAb therapy (11).

This is of particular importance for patients undergoing resection of primary colorectal cancer (CRC). Approximately 1.2 million patients worldwide are diagnosed with CRC annually, and approximately 600,000 patients die from this disease each year (26). Surgical removal of the primary tumor is the preferred therapy, as it is the only treatment that can provide long-term disease-free survival. Unfortunately, development of liver metastases is a frequent complication and accompanied by high mor-

duction by macrophages was blocked by incubation with EDA in live-cell experiments (Figure 7, A and B). However, degradation seemed to be somewhat less efficient. We therefore used ImageStream technology to quantify uptake and breakdown of tumor cells in the presence of EDA, as multiple images of single cells in flow can be acquired with this novel flow cytometry technique. No difference was observed in the number of macrophages that had phagocytosed anti-EGFR mAb-opsonized A431 cells in the presence or absence of EDA after 4 hours (Figure 7C). This supports that ROS are not necessary for ADPh. Phagocytosed tumor cells within macrophages were smaller compared with nonphagocytosed tumor cells, which is indicative of breakdown (Figure 7, D-F). A small, albeit not significantly different, increase in size of tumor cells was observed in macrophages that had been treated bidity and mortality (27). Many patients develop liver metastases after resection of colon carcinoma, despite lack of evidence of metastatic disease at the time of resection of the primary tumor, which indicates that surgery could either inadvertently release malignant cells into blood or induce circulating malignant cells to adhere in the liver. Disseminated tumor cells can be detected in the blood of the majority of patients at the time of surgery (28). Furthermore, a recent meta-analysis unequivocally demonstrated that the presence of circulating tumor cells at the time of surgery correlates with poor prognosis of patients with CRC (29). Paradoxically, resection of the tumor contributes to the risk of metastases development, as it creates permissive circumstances for tumor cells to adhere in the liver $(20,21)$. Kupffer cells reside in the liver sinusoids and clear the portal blood circulation of bacteria, old blood cells, or foreign substances. 
As such, they are the first line of defense against tumor cells entering the liver (30). We now show that Kupffer cells were not very effective in arrest of circulating tumor cells in the absence of mAbs, even though they were able to sample small particles of tumor cells. Still, these results explained why colocalization between Kupffer cells and tumor cells was observed in previous immunohistochemistry experiments (19). By contrast, after mAb therapy, they efficiently phagocytosed intact tumor cells, which prevented liver metastases development. Thus, patients who are at risk of developing liver metastases, such as patients undergoing resection of CRC, may greatly benefit from preoperative $\mathrm{mAb}$ adjuvant therapy.

Additionally, macrophages may play a major role in successes of $\mathrm{mAb}$ therapy in patients with hematological malignancies. It was demonstrated that patients with an Fc $\gamma$ RIIIa-158V allotype had better clinical responses after rituximab therapy, compared with patients with the FcyRIIIa-158F allotype (31). This effect was mainly attributed to FcyRIII-expressing NK cells (15). However, because human macrophages express FcrRIII (32), we hypothesize that the difference in therapeutic efficacy of mAbs may be partly attributed to phagocytosis by macrophages in addition to NK cell-mediated ADCC, particularly because macrophages proved extremely efficient in phagocytosing malignant B cells in the presence of anti-CD20 mAb (data not shown). Furthermore, macrophage depletion abrogated therapeutic efficacy of anti-CD20 mAb in a murine lymphoma model $(25,33)$. Similarly, SCID mice that had been engrafted with a Hodgkin-derived cell line and were treated with either antiCD30 or anti-CD40 mAbs had worse survival if macrophages had been depleted. Removal of NK cells or neutrophils did not significantly influence therapeutic efficacy $(34,35)$. These latter results also support the view that direct killing by antibody-induced apoptosis or CDC are less likely mechanisms of action. Interestingly, clinical responses to rituximab therapy have also been correlated with polymorphisms in human FcyRIIa (14). Since macrophages, but not NK cells, express Fc $\gamma$ RII, a role for macrophages as effector cells in B cell lymphoma depletion after anti-CD20 mAb treatment of patients is strongly supported.

It is less clear whether macrophages also contribute to tumor cell killing in solid malignancies. In our experiments, $\mathrm{mAb}$ therapy was ineffective when given after the establishment of micrometastases in the liver. Intravital microscopy demonstrated that Kupffer cells were stationary and not recruited, arguing against an important role for Kupffer cells in mAb therapy of existing liver metastases. Moreover, it was shown that Kupffer cells can suppress T cell effector function. For instance, Kupffer cells in hepatocellular carcinoma had higher expression of the B7-H1 molecule, which suppressed $\mathrm{CD}^{+} \mathrm{T}$ cell function through interaction with programmed death- 1 on $\mathrm{CD}^{+} \mathrm{T}$ cells $(36,37)$. Additionally, galactin-9 expression on Kupffer cells induced $\mathrm{T}$ cell senescence via Tim-3 signaling in hepatitis B virus-associated hepatocellular carcinoma (38). mAb therapy likely will not overcome Kupffer cell-associated immune escape in existing tumors, and this is supported by the fact that anti-EGFR mAbs are only indicated for the treatment of metastatic colorectal cancers that express wild-type KRAS, as this therapy is ineffective when tumors harbor a KRAS mutation (6). This suggests that, in established tumors, direct effects of anti-EGFR mAbs are more prominent than Fc $\gamma$ receptor-mediated effector mechanisms. However, since we are proposing to prevent the formation of metastasis during surgery by delivery of antibody prior to metastasis formation, this may be a valuable clinical approach.
Interestingly, tumor-associated macrophages that were isolated from mouse breast carcinomas expressed $\mathrm{Fc} \gamma$ receptors and were capable of phagocytosing breast carcinoma cells in the presence of anti-CD142 mAbs (39). Furthermore, depletion of macrophages reduced the success of anti-CD142 mAb therapy to prevent breast carcinoma outgrowth and metastasis in mice, suggesting that macrophages may play an important role in therapeutic efficacy of $\mathrm{mAb}$ therapy in breast cancer. It was demonstrated that macrophages in most malignancies, including ovarian cancer and breast carcinoma, have an alternatively activated M2 phenotype and are considered protumorigenic, as they secrete growth and angiogenic factors as well as immunosuppressive factors (40). Nonetheless, anti-CD142 mAb therapy was able to repolarize macrophages into potent cytotoxic effector cells in breast carcinoma (39).

The mechanism(s) of macrophage-induced killing of tumor cells has not yet been completely elucidated. Recently, it was suggested that macrophages mainly eliminate tumor cells via ADCC, as synapse formation between tumor cells and macrophages was observed in peritoneal lavages of mAb-treated mice (24). As uptake of tumor cells by macrophages requires close interactions, synapse formation is likely involved. However, we now show that the main mechanism by which Kupffer cells eliminate tumor cells in vivo is through ADPh. Furthermore, both murine bone marrow-derived and human monocyte-derived macrophages killed a wide variety of mouse and human tumor cells with different target antigens on colon carcinoma (EGFR), vulvar carcinoma (EGFR), or B cell lymphoma (CD20) via $\mathrm{ADPh}$, as shown in live-cell imaging experiments (data not shown). Additionally, production of cytotoxic ROS and RNS has been proposed as a major killing mechanism by macrophages (41). In this study, ADPh was independent of ROS and RNS production. Moreover, ROS inhibition neither interfered with acidification of phagolysosomes, nor interfered with intracellular breakdown of tumor cells. Thus, intracellular digestion in lysosomes was the main mechanism of tumor cell killing.

Taken together, Kupffer cells were essential in eliminating circulating tumor cells after mAb immunotherapy. These findings may contribute significantly to the design of optimized $\mathrm{mAb}$ therapeutical strategies of many patients with cancer by helping us to promote recruitment of macrophages as effector cells. Macrophages may especially play a prominent role in $\mathrm{mAb}$-mediated eradication of tumor cells in patients with hematological malignancies. Moreover, we propose that patients undergoing resection for primary CRC may greatly benefit from preoperative $\mathrm{mAb}$ immunotherapy, as this will lead to elimination of remaining circulating tumor cells by Kupffer cells and may prevent the development of postsurgical liver metastases.

\section{Methods}

\section{Animal models}

Wild-type C57BL/6 and Balb/c mice were purchased from Charles River. Fc $\gamma$ RI-deficient mice (42) were provided by T.W. Du Clos (University of New Mexico, Albuquerque, New Mexico, USA) and S.J. Verbeek (Leiden University Medical Center, Leiden, The Netherlands). All mice had access to food and water ad libitum and were housed in specific pathogen-free, double-barrier units at the University of Calgary or VU University Medical Center. Animals were used between 8 and 12 weeks of age.

\section{Cell culture}

Cell lines. The mouse melanoma cell line B16F10 (ATCC), expressing $\mathrm{gp} 75$, was cultured as described previously (11). As the gp75 protein is 
mostly expressed intracellularly due to an intracellular retention motive (NQPLLTD) (43), B16F10 cells were transfected with gp75 lacking this motive for in vitro experiments. A HindIII/EcoRI fragment containing the coding sequence of mGP75 (accession no. NM_031202), but with only the first 10 membrane-proximal amino acids of the $\mathrm{C}$ terminus (RSRSTKNEAN), was used, as this lacks the retention signal (43). This fragment was subcloned into pcDNA3.1 and transfected using Lipofectamine LTX according to the manufacturers' instructions (Invitrogen). After selection with $200 \mu \mathrm{g} / \mathrm{ml}$ Zeocin (Sigma-Aldrich) for 7 days, cells staining positive for membrane gp75 were sorted using mouse IgG2a TA99 mAb (produced as described previously in ref. 11) and goat antimouse IgG-FITC (Sanquin).

Mouse C26 colon carcinoma cells (ATCC) were grown to subconfluency in 6-well plates and transfected with $1 \mu \mathrm{g}$ human EGFR construct (hEGFR in USE/amp/neo vector, Upstate Technology) using the Fugene-6 transfection system (Roche Applied Science), according to the manufacturer's instructions. Positive cells were selected 48 hours after transfection by incubating cells with $3.5 \mu \mathrm{g} / \mathrm{ml} \mathrm{G} 418$ in DMEM medium supplemented with $10 \%$ heat-inactivated FCS, penicillin $(100 \mathrm{U} / \mathrm{ml})$, streptomycin $(100 \mu \mathrm{g} / \mathrm{ml})$, and L-glutamine $(200 \mu \mathrm{M}$; referred to below as complete DMEM). The human vulvar carcinoma cell line A431 (ATCC) was cultured under standard conditions in complete DMEM.

For in vivo or in vitro experiments, single cell suspensions $\left(5 \times 10^{6}\right.$ cells per $\mathrm{ml}$ ) were prepared by enzymatic detachment using trypsin-EDTA solution (Invitrogen). Viability was assessed by trypan blue exclusion and always exceeded 95\%. Cell suspensions were fluorescently labeled by incubating cells in complete DMEM containing $50 \mu \mathrm{g} / \mathrm{ml}$ DiI or DiB (Sigma-Aldrich) according to the manufacturer's instructions.

Murine bone marrow macrophages. Bone marrow from wild-type C57BL/6 or Balb/c mice was harvested from freshly isolated femurs, tibiae, and humeri. After removal of connective tissues and muscles, bone marrow was flushed and single cell suspensions were made by passing bone marrow through a sterile $70-\mu \mathrm{m}$ filter (BD Falcon). Macrophages were differentiated by incubating bone marrow cells for 7 days with complete DMEM, supplemented with 15\% L929-conditioned medium (containing M-CSF). Macrophages were harvested after 15 -minute incubation with trypsinEDTA and subsequent scraping using a cell scraper. For live-cell imaging, macrophages were seeded in 8 -well ibiTreat $\mu$-Slides $\left(2 \times 10^{5}\right.$ cells per well; ibidi). For in vitro phagocytosis experiments, macrophages were seeded in 6-well culture plates.

\section{In vivo experiments}

Intravital microscopy of the liver. Wild-type or Fc $\gamma \mathrm{RI}$-deficient mice were injected i.p. with vehicle (PBS), isotype control (MG-4), or $200 \mu \mathrm{g}$ TA99. After 24 hours mice were anesthetized by i.p. injection of a mixture of 10 $\mathrm{mg} / \mathrm{kg}$ xylazine and $200 \mathrm{mg} / \mathrm{kg}$ ketamine hydrochloride (Bimeda-MTC). Body temperature was maintained at $37^{\circ} \mathrm{C}$ using an infrared heat lamp. The right vena jugularis was cannulated for i.v. administration of antibodies and additional anesthetic as required. Alexa Fluor 647-labeled F4/80 (eBioscience) antibody was injected i.v. to stain Kupffer cells. A small incision was made in the left flank to reveal the spleen. $2 \times 10^{5}$ DiI-labeled B16F10 cells were injected intrasplenically at a constant rate to allow flow of tumor cells toward the liver. After 1 minute, splenectomy was performed. Additionally, FcyRI-deficient mice were injected with the FcrRIV blocking antibody 9E9 (44) (a gift of F. Nimmerjahn, University of Erlangen-Nuremberg, Erlangen, Germany) 1 hour prior to tumor cell injections. Alternatively, mice were treated by i.p. injection of $150 \mathrm{mg} / \mathrm{kg}$ ROS scavenger EDA $(20,45)$ and/or $10 \mathrm{mg} / \mathrm{kg} \mathrm{NO}$ synthesis inhibitor L-NMMA (46) 1 hour and/or 30 minutes prior to tumor cell injection. For visualization of ROS production, animals were injected with $10 \mu \mathrm{g}$ CM-H2DCFDA (Molecular Probes) prior to injection of tumor cells. The livers of mice were prepared for in vivo microscopic observation as previously described (47).

Alternatively, Kupffer cells were depleted by injection of clodronate liposomes 1 day before injection of tumor cells $(19,22)$. DiI-labeled C26hEGFR cells $\left(10^{6}\right.$ cells per $\left.\mathrm{ml}\right)$ were incubated with 1:100 diluted Cetuximab (anti-hEGFR mAb; Merck Serono) or vehicle for 30 minutes on ice, after which cells were extensively washed. $2 \times 10^{5}$ cells were injected into the spleens of control or Kupffer cell-depleted mice, and splenectomy was performed. Livers of animals were investigated at $0,1,4$, and 5 days after injection of tumor cells. The livers were visualized by i.v. injection of Alexa Fluor 647-labeled CD31 antibody (BD Bioscience) to stain blood vessels. Kupffer cells were stained by injection of Alexa Fluor 750-labeled F4/80 (eBioscience) antibody. Twenty representative images were taken from the liver of each mouse for quantitative analysis.

Distribution of tumor cells after Kupffer cell depletion was investigated in control or Kupffer cell-depleted mice by performing midline laparotomy under anesthesia, and DiI-labeled B16F10gp75 cells $\left(2.5 \times 10^{6}\right.$ cells $)$ were injected in a mesenteric vein. Animals were sacrificed 45 minutes after injection of tumor cells by cardiac puncture. Tissue samples from hearts, lungs, spleens, and kidneys were frozen for microscopic analyses. The number of DiI-labeled B16F10-gp75 cells in blood was measured by flow cytometry with Cyan ADP High Performance Research Flow Cytometer (Beckman Coulter Inc.)

Liver metastases development. The effect of TA99 treatment on tumor development in control and Kupffer cell-depleted mice was studied by depletion of Kupffer cells with clodronate liposomes as described previously (22). All animals were injected i.p. on days 0, 2, and 4 with $200 \mu \mathrm{g}$ TA99 or MG-4 as an irrelevant isotype control mAb in PBS. Tumor cells were injected intrasplenically, and splenectomy was performed (11). After 3 weeks, mice were sacrificed, and the number of liver metastases in each mouse was scored.

To investigate the therapeutic effect of anti-EGFR mAbs in existing liver metastases, mice were injected with C26-hEGFR cells at day 0. After day 5, mice received i.p. injections of $200 \mu \mathrm{g}$ anti-hEGFR mAb every 48 hours. At day 10 mice were injected i.v. with FITC-albumin for visualization of liver tissue. High fluorescence indicates healthy liver tissue, as visualized by FITC-albumin, whereas dark areas with abnormal tissue structure are indicative of tumor, because of poor perfusion and penetration of FITC-albumin into tumor tissue. Livers were visualized, and 20 stitched field pictures were taken for quantification.

\section{Fluorescence microscopy}

Cryostat tissue sections were fixed for 10 minutes in acetone and air dried. After blocking with $10 \%$ normal goat serum, sections were incubated with 1:100 rat anti-mouse F4/80 mAbs for 1 hour at room temperature. After washing, visualization was achieved by incubating the sections with 1:300 goat anti-rat Alexa Fluor 488 (Molecular Probes) for 30 minutes at room temperature. Cell nuclei were stained with Hoechst (Molecular Probes). Sections were washed, mounted, and examined with a LeicaDM6000 fluorescence microscope (Leica Microsystems).

\section{In vitro cytotoxicity assays}

Live-cell imaging. Macrophages were labeled fluorescently with DiO, DiI, or LysoID Red (Enzo Life Sciences). DiI- or DiB-labeled B16F10-gp75, C26-hEGFR, or A431 cells were added in an $\mathrm{E} / \mathrm{T}$ ratio of 15:1 with TA99 mAbs, anti-EGFR mAbs, or MG-4 (isotype) antibodies. Real-time phagocytosis assays were performed with an Olympus CellR live-imaging station (type IX81, UPLFLN 40xO/1.3 lens). Images were taken every 5 minutes with an Olympus ColorView II camera for 3 to 6 hours, followed by a 15-minute interval for 18 to 21 hours. 
Flow cytometry and AMNIS. Tumor cells were fluorescently labeled with $\mathrm{DiB}$ and added to macrophage cultures in presence or absence of tumor-specific mAbs. Cocultures were incubated at $37^{\circ} \mathrm{C}$ for 1 or 4 hours. To investigate the effect of ROS scavenging on phagocytosis of tumor cells, macrophages were incubated with $1 \mu \mathrm{M}$ EDA for 1 hour before addition of tumor cells in a culture stove. To confirm that EDA inhibited ROS production by macrophages, cells were incubated with $50 \mathrm{~g} / \mathrm{ml}$ peptidoglycan from S. aureus (InvivoGen) for 1 hour. After harvesting of cells, PE-labeled F4/80 antibody (Molecular Probes) or $10 \mu \mathrm{M}$ CM-H2DCFDA (Molecular Probes) was added for detection of macrophages or intracellular ROS levels, respectively. After an incubation of 30 minutes on ice, cells were washed extensively. Measurements were performed by using Cyan ADP High Performance Research Flow Cytometer (Beckman Coulter Inc.) or ImageStreamX (Amnis Corporation) flow cytometers.

\section{Quantifications}

In vivo. Contact between Kupffer cells and tumor cells was defined as interaction between these two cells types, whereas phagocytosis of tumor cells by Kupffer cells in the livers was determined as colocalization of complete tumor cells within Kupffer cells. Distribution of tumor cells into organs was quantified by counting the number of tumor cells per image (5 random images per organ per mouse). Differences between treatments were quantified in at least 6 independent experiments with AnalySIS software (SoftImagingSystem $\mathrm{GmbH}$ ). The number of micrometastases was determined by counting, whereas the total area was determined by measuring red area in images (indicating the presence of DiI-labeled tumor cells). Metastatic burden was calculated by dividing area of metastases $\left(\mathrm{mm}^{2}\right)$ by area of liver from which the images were taken $\left(\mathrm{mm}^{2}\right)$.

In vitro phagocytosis of DiB-labeled tumor cells by macrophages (stained with F480-PE) and intracellular degradation was evaluated with imaging flow cytometry (ImageStreamX). The ImageStream ISX from AMNIS is an instrument that combines flow cytometry and microscopy in one platform. Briefly, bright-field and fluorescence images were acquired for a minimum of 5,000 cells per sample. Using the IDEAS analysis software (AMNIS), a compensation matrix was generated and applied to the acquired images. Single cells were selected with a bivariate plot for the area and aspect ratio features of the bright-field images. Single cells with a bright-field gradient RMS value of 55 or higher were considered to be in focus. Macrophage/tumor cell complexes were selected from single/focused cells as the double-positive population within a bivariate plot for the PE intensity and DiB intensity. Macrophages were scored as positive for tumor cell uptake if the internalization value of the DiB was 4 or higher within the PE morphology mask. Tumor cell degradation was determined by applying - to macrophages with tumor cell uptake - the spot count feature on DiB objects with a threshold mask of 90 .

\section{Statistics}

For comparisons between 2 groups, 2-tailed Student's $t$ test was used. For comparisons among more than 2 groups, 2-way ANOVA was used. Statistical significance was accepted at $P<0.05$. Results are presented as mean $\pm \mathrm{SEM}$.

\section{Study approval}

Animal experimental protocols were approved by the Animal Care Committee of the University of Calgary and the Canadian Council on the Use of Laboratory Animals in Calgary, Canada, or the Committee for Animal Research of the VU University Medical Center in Amsterdam in accordance with all institutional and national guidelines.

\section{Acknowledgments}

The authors would like to thank T. O'Toole for expert help with AMNIS experiments and N. Blijleven and M.W.M. van Hout for support with information technology. This project was financially supported by the Dutch Cancer Foundation (KWF) project VU2011-4931.

Received for publication September 11, 2012, and accepted in revised form November 7, 2013.

Address correspondence to: Marjolein van Egmond, Department of Molecular Cell Biology and Immunology, Van der Boechorststraat 7 J295, 1081 BT Amsterdam, The Netherlands. Phone: 31.0.20.444.59.75; Fax: 31.0.20.444.80.81; E-mail: m.vanegmond@vumc.nl.
1. Scott AM, Wolchok JD, Old LJ. Antibody therapy of cancer. Nat Rev Cancer. 2012;12(4):278-287.

2. Weiner LM, Surana R, Wang S. Monoclonal antibodies: versatile platforms for cancer immunotherapy. Nat Rev Immunol. 2010;10(5):317-327.

3. Amoroso A, et al. Understanding rituximab function and resistance: implications for tailored therapy. Front Biosci. 2011;16:770-782.

4. Hogarth PM, Pietersz GA. Fc receptor-targeted therapies for the treatment of inflammation, cancer and beyond. Nat Rev Drug Discov. 2012;11(4):311-331.

5. Weiner LM, Murray JC, Shuptrine CW. Antibody-based immunotherapy of cancer. Cell. 2012; 148(6):1081-1084.

6 . Benvenuti $S$, et al. Oncogenic activation of the RAS/RAF signaling pathway impairs the response of metastatic colorectal cancers to anti-epidermal growth factor receptor antibody therapies. Cancer Res. 2007;67(6):2643-2648

7. Racila E, et al. A polymorphism in the complement component $\mathrm{C} 1 \mathrm{qA}$ correlates with prolonged response following rituximab therapy of follicular lymphoma. Clin Cancer Res. 2008;14(20):6697-6703.

8. Bevaart L, Jansen MJ, van Vugt MJ, Verbeek JS, van de Winkel JG, Leusen JH. The high-affinity IgG receptor, Fc $\gamma$ RI, plays a central role in antibody therapy of experimental melanoma. Cancer Res. 2006;66(3):1261-1264.

9. Clynes RA, Towers TL, Presta LG, Ravetch JV. Inhibitory $\mathrm{Fc}$ receptors modulate in vivo cytotoxicity against tumor targets. Nat Med. 2000;6(4):443-446.

10. Nimmerjahn F, Ravetch JV. Divergent immunoglobulin g subclass activity through selective Fc receptor binding. Science. 2005;310(5753):1510-1512.

11. Otten MA, et al. Experimental antibody therapy of liver metastases reveals functional redundancy between Fc $\gamma$ RI and Fc $\gamma$ RIV. J Immunol. 2008; 181(10):6829-6836.

12. Bibeau F, et al. Impact of Fc $\gamma$ RIIa-Fc $\gamma$ RIIIa polymorphisms and KRAS mutations on the clinical outcome of patients with metastatic colorectal cancer treated with cetuximab plus irinotecan. J Clin Oncol. 2009;27:1122-1129.

13. Musolino A, et al. Immunoglobulin G fragment $\mathrm{C}$ receptor polymorphisms and clinical efficacy of trastuzumab-based therapy in patients with HER-2/neu-positive metastatic breast cancer. J Clin Oncol. 2008;26:1789-1796.

14. Weng WK, Levy R. Two immunoglobulin G fragment $\mathrm{C}$ receptor polymorphisms independently predict response to rituximab in patients with follicular lymphoma. J Clin Oncol. 2003;21:3940-3947.

15. Hatjiharissi E, et al. Increased natural killer cell expression of CD16, augmented binding and ADCC activity to rituximab among individuals expressing the FcyRIIIa-158 V/V and V/F polymorphism. Blood. 2007;110(7):2561-2564.

16. Bilitewski U. Determination of immunomodulatory effects: focus on functional analysis of phagocytes as representatives of the innate immune sys- tem. Anal Bioanal Chem. 2008;391(5):1545-1554.

17. Braster R, O’Toole T, van Egmond M. Myeloid cells as effector cells for monoclonal antibody therapy of cancer. Methods. 2013; pii:S1046-2023(13)00221-1.

18. Albanesi M, et al. Neutrophils mediate antibody-induced anti-tumor effects in mice. Blood. 2013; 122(18):3160-3164.

19. van der Bij GJ, et al. Experimentally induced liver metastases from colorectal cancer can be prevented by mononuclear phagocyte-mediated monoclonal antibody therapy. J Hepatol. 2010;53(4):677-685.

20. Gul N, et al. Surgery-induced reactive oxygen species enhance colon carcinoma cell binding by disrupting the liver endothelial cell lining. Gut. 2011;60(8):1076-1086.

21. van der Bij GJ, et al. Blocking alpha2 integrins on rat CC531s colon carcinoma cells prevents operation-induced augmentation of liver metastases outgrowth. Hepatology. 2008;47(2):532-543.

22. van Rooijen N, Sanders A. Liposome mediated depletion of macrophages: mechanism of action, preparation of liposomes and applications. J Immunol Methods. 1994;174(1-2):83-93.

23. Perez R, Crombet T, de LJ, Moreno E. A view on EGFR-targeted therapies from the oncogene-addiction perspective. Front Pharmacol. 2013;4:53.

24. Hubert P, et al. Antibody-dependent cell cytotoxicity synapses form in mice during tumor-specific antibody immunotherapy. Cancer Res. 2011;71(15):5134-5143.

25. Minard-Colin V, et al. Lymphoma depletion dur- 
ing CD20 immunotherapy in mice is mediated by macrophage Fc $\gamma$ RI, Fc $\gamma$ RIII, and Fc $\gamma$ RIV. Blood. 2008;112(4):1205-1213.

26. Jemal A, Bray F, Center MM, Ferlay J, Ward E, Forman D. Global cancer statistics. CA Cancer J Clin. 2011;61(2):69-90.

27. Siegel R, et al. Cancer treatment and survivorship statistics, 2012. CA Cancer JClin. 2012;62(4):220-241.

28. Wind J, et al. Circulating tumour cells during laparoscopic and open surgery for primary colonic cancer in portal and peripheral blood. Eur J Surg Oncol. 2009;35(9):942-950.

29. Rahbari NN, et al. Meta-analysis shows that detection of circulating tumor cells indicates poor prognosis in patients with colorectal cancer. Gastroenterology. 2010;138(5):1714-1726.

30. Paschos KA, Majeed AW, Bird NC. Role of Kupffer cells in the outgrowth of colorectal cancer liver metastases. Hepatol Res. 2010;40(1):83-94.

31. Persky DO, et al. $F_{C} \gamma$ receptor 3 a genotype predicts overall survival in follicular lymphoma patients treated on SWOG trials with combined monoclonal antibody plus chemotherapy but not chemotherapy alone. Haematologica. 2012;97(6):937-942.

32. Nimmerjahn F, Ravetch JV. Antibodies, Fc receptors and cancer. Curr Opin Immunol. 2007;19(2):239-245.

33. Horikawa M, Minard-Colin V, Matsushita T, Tedder TF. Regulatory B cell production of IL-10 inhibits lymphoma depletion during CD20 immunotherapy in mice. J Clin Invest. 2011;121(11):4268-4280.

34. Oflazoglu E, et al. Macrophages contribute to the antitumor activity of the anti-CD30 antibody SGN-30. Blood. 2007;110(13):4370-4372.

35. Oflazoglu E, et al. Macrophages and Fc-receptor interactions contribute to the antitumour activities of the anti-CD40 antibody SGN-40. BrJ Cancer. 2009;100(1):113-117.

36. Kryczek I, et al. B7-H4 expression identifies a novel suppressive macrophage population in human ovarian carcinoma. J Exp Med. 2006;203(4):871-881.

37. Wu K, Kryczek I, Chen L, Zou W, Welling TH Kupffer cell suppression of CD8+ T cells in human hepatocellular carcinoma is mediated by B7-H1/ programmed death-1 interactions. Cancer Res. 2009;69(20):8067-8075

38. Li H, et al. Tim-3/galectin-9 signaling pathway mediates T-cell dysfunction and predicts poor prognosis in patients with hepatitis B virus-associated hepatocellular carcinoma. Hepatology. 2012;56(4):1342-1351.

39. Grugan KD, et al. Tumor-associated macrophages promote invasion while retaining Fcdependent anti-tumor function. J Immunol. 2012; 189(11):5457-5466.

40. Mantovani A, Sica A. Macrophages, innate immunity and cancer: balance, tolerance, and diversity. Curr Opin Immunol. 2010;22(2):231-237.
41. Forman HJ, Torres M. Redox signaling in macrophages. Mol Aspects Med. 2001;22(4-5):189-216.

42. Ioan-Facsinay A, et al. Fc $\gamma$ RI (CD64) contributes substantially to severity of arthritis, hypersensitivity responses, and protection from bacterial infection. Immunity. 2002;16(3):391-402.

43. Vijayasaradhi S, Xu Y, Bouchard B, Houghton AN Intracellular sorting and targeting of melanosomal membrane proteins: identification of signals for sorting of the human brown locus protein, gp75. J Cell Biol. 1995;130(4):807-820.

44. Nimmerjahn F, Bruhns P, Horiuchi K, Ravetch JV. FcyRIV: a novel FcR with distinct IgG subclass specificity. Immunity. 2005;23(1):41-51.

45. Tajima $S$, et al. Preventive effects of edaravone, a free radical scavenger, on lipopolysaccharide-induced lung injury in mice. Respirology. 2008;13(5):646-653.

46. Cunha TM, et al. Stimulation of peripheral $\kappa$ opioid receptors inhibits inflammatory hyperalgesia via activation of the PI3K $\gamma / \mathrm{AKT} / \mathrm{nNOS} / \mathrm{NO}$ signaling pathway. Mol Pain. 2012;8:10.

47. Wong CH, Jenne CN, Lee WY, Leger C, Kubes P. Functional innervation of hepatic iNKT cells is immunosuppressive following stroke. Science. 2011;334(6052):101-105.

48. Davies LC, Jenkins SJ, Allen JE, Taylor PR. Tissue-resident macrophages. Nat Immunol. 2013; 14(10):986-995 